\title{
CERTAIN GEOMETRIC PROPERTIES OF A NORMALIZED HYPER-BESSEL FUNCTION
}

\author{
İbrahim Aktaş
}

\begin{abstract}
(c) 2020 by University of Niš, Serbia | Creative Commons License: CC BY-NC-ND
\end{abstract}
\begin{abstract}
In the present paper, by making use of previous results on analytic functions, certain geometric properties including starlikeness of order $\alpha$ and convexity of order $\alpha$ of a normalized hyper-Bessel function have been investigated. In addition, some conditions of the mentioned function which belongs to the Hardy space have been given. Moreover, specific examples of the results which refer to special values of the parameters have also been presented.
\end{abstract}

Keywords: analytic function, hyper-Bessel function, starlikeness of order $\alpha$, convexity of order $\alpha$, Hardy space.

\section{Introduction}

Special functions have a great importance in applied sciences. Due to their remarkable properties, a great number of mathematicians have investigated the geometric properties of special functions like Bessel, Struve and Lommel functions of the first kind. Especially, these geometric properties include univalence, starlikeness and convexity of the above mentioned functions. Actually, the first results on the univalence of Bessel function can be found in the papers [9] and [13] written by Brown and, Kreyszig and Todd in 1960, respectively. In recent years, the geometric properties of some special functions have become very interesting to mathematicians. More precisely, the authors in $[3,4,5,6]$ began to study the geometric properties of the above mentioned special functions and their generalizations. Also, the authors in $[7,14]$ studied Hardy space of generalized Bessel and hypergeometric functions, respectively. Especially, Bessel function has some extensions like $q$-Bessel and hyper-Bessel functions. Some geometric properties of these extensions may be found in $[1,2,3]$ and the references therein.

Now, we would like to remind you about the definitions of the Bessel and hyperBessel functions, respectively. Bessel function is defined by the following infinite

Received February 22, 2019; accepted May 16, 2019

2010 Mathematics Subject Classification. Primary 30C45; Secondary 33E20, 33E50 
series (see [16]):

$$
J_{\nu}(z)=\sum_{n \geq 0} \frac{(-1)^{n}}{n ! \Gamma(\nu+n+1)}\left(\frac{z}{2}\right)^{2 n+\nu}, z \in \mathbb{C}
$$

where $\Gamma(z)$ denotes the familiar gamma function. Similarly, the hyper-Bessel function is defined by (see [10]):

$$
J_{\gamma_{d}}(z)=\sum_{n \geq 0} \frac{(-1)^{n}\left(\frac{z}{d+1}\right)^{n(d+1)+\sum_{i=1}^{d} \gamma_{i}}}{n ! \prod_{i=1}^{d} \Gamma\left(\gamma_{i}+n+1\right)} .
$$

It is clear that, the hyper-Bessel function is reduced to the classical Bessel function given by (1.1) for $d=1$ and $\gamma_{1}=\nu$. Due to this relationship, the earlier studies on the classical Bessel function can be extended to the hyper-Bessel function. Motivated by some earlier works, our main aim in this study is to obtain new conditions on the starlikeness and convexity of the hyper-Bessel functions. Also, we will deal with the Hardy space of the hyper-Bessel function.

\section{Starlikeness and convexity of hyper-Bessel function}

In the beginning of this section, we are going to mention some basic notions in geometric function theory. Let $\mathbb{D}_{r}$ be the open disk $\{z \in \mathbb{C}:|z|<r\}$ with radius $r>0$ and $\mathbb{D}_{1}=\mathbb{D}$. Let $\mathcal{A}$ denote the class of analytic functions $f: \mathbb{D}_{r} \rightarrow \mathbb{C}$,

$$
f(z)=z+\sum_{n \geq 2} a_{n} z^{n}
$$

which satisfies the normalization conditions $f(0)=f^{\prime}(0)-1=0$. By $\mathcal{S}$ we mean the class of functions belonging to $\mathcal{A}$ which are univalent in $\mathbb{D}_{r}$. Also, for $0 \leq \alpha<1$, by $\mathcal{S}^{\star}(\alpha)$ and $\mathcal{C}(\alpha)$ we will denote the subclasses of $\mathcal{A}$ consisting of functions which are starlike and convex of order $\alpha$, respectively. The analytic characterizations of these subclasses are

$$
\mathcal{S}^{\star}(\alpha)=\left\{f: f \in \mathcal{A} \text { and } \operatorname{Re}\left(\frac{z f^{\prime}(z)}{f(z)}\right)>\alpha \text { for } z \in \mathbb{D}\right\}
$$

and

$$
\mathcal{C}(\alpha)=\left\{f: f \in \mathcal{A} \text { and } \operatorname{Re}\left(1+\frac{z f^{\prime \prime}(z)}{f^{\prime}(z)}\right)>\alpha \text { for } z \in \mathbb{D}\right\},
$$

respectively.

The following results on analytic functions given by Silverman in [15] will be used in order to prove our main results. 
Lemma 2.1. Let $f$ is of the form (2.1). If

$$
\sum_{n=2}^{\infty}(n-\alpha)\left|a_{n}\right| \leq 1-\alpha
$$

then $f \in \mathcal{S}^{\star}(\alpha)$.

Lemma 2.2. Let $f$ is of the form (2.1). If

$$
\sum_{n=2}^{\infty} n(n-\alpha)\left|a_{n}\right| \leq 1-\alpha
$$

then $f \in \mathcal{C}(\alpha)$.

Before revealing our main results concerning starlikeness and convexity of the hyper-Bessel function, we will apply the following natural normalization for the function $z \mapsto J_{\gamma_{d}}(z)$ given by (1.2):

$$
\begin{aligned}
F_{\gamma_{d}}(z) & =z \frac{\prod_{i=1}^{d} \Gamma\left(\gamma_{i}+1\right)}{\left(\frac{d+1}{z}\right)^{\sum_{i=1}^{d} \gamma_{i}}} J_{\gamma_{d}}(\sqrt[d+1]{z}) \\
& =z+\sum_{n \geq 2} \frac{(-1)^{n}}{(n-1) ! \rho^{n-1} \prod_{i=1}^{d}\left(\gamma_{i}+1\right)_{n-1}} z^{n},
\end{aligned}
$$

where $\rho=(d+1)^{d+1}$ and $(\beta)_{n}$ is the Pochhammer symbol which is defined by $(\beta)_{0}=1$ and $(\beta)_{n}=\beta(\beta+1) \ldots(\beta+n-1)$ for $n \geq 1$. As a consequence, the function $z \mapsto F_{\gamma_{d}} \in \mathcal{A}$.

Our first main result regarding starlikeness of order $\alpha$ of the function $F_{\gamma_{d}}(z)$ is the following:

Theorem 2.1. Let $\alpha \in[0,1), d=1,2, \ldots, \rho=(d+1)^{d+1}$ and $\mu=\prod_{i=1}^{d}\left(\gamma_{i}+1\right)$. If

$$
\alpha \leq \frac{4 \rho^{2} \mu^{2}-12 \rho \mu+3}{4 \rho^{2} \mu^{2}-8 \rho \mu+3},
$$

then the normalized hyper-Bessel function $F_{\gamma_{d}}(z)$ is starlike of order $\alpha$ in $\mathbb{D}$.

Proof. It is known from Lemma 2.1 that, if the function $f \in \mathcal{A}$ satisfies the inequality (2.2), then the function $f$ is starlike of order $\alpha$. Because of this, in order to prove starlikeness of order $\alpha$ of the function $F_{\gamma_{d}}(z)$, it is enough to show that

$$
\sum_{n \geq 2}(n-\alpha)\left|\frac{(-1)^{n-1}}{(n-1) ! \rho^{n-1} \prod_{i=1}^{d}\left(\gamma_{i}+1\right)_{n-1}}\right| \leq 1-\alpha
$$

for $\alpha \in[0,1)$. Now using the well-known inequalities

$$
2^{n-2} \leq(n-1) !
$$


and

$$
(\beta)_{n} \leq(\beta)^{n}
$$

for $n \geq 2$, we can write that

$$
\begin{aligned}
\sum_{n \geq 2}(n-\alpha)\left|\frac{(-1)^{n-1}}{(n-1) ! \rho^{n-1} \prod_{i=1}^{d}\left(\gamma_{i}+1\right)_{n-1}}\right| & =\sum_{n \geq 2} \frac{(n-\alpha)}{(n-1) ! \rho^{n-1} \prod_{i=1}^{d}\left(\gamma_{i}+1\right)_{n-1}} \\
& \leq 2 \sum_{n \geq 2} \frac{n-\alpha}{(2 \rho \mu)^{n-1}} \\
& =2 \sum_{n \geq 2} n\left(\frac{1}{2 \rho \mu}\right)^{n-1}-2 \alpha \sum_{n \geq 2}\left(\frac{1}{2 \rho \mu}\right)^{n-1}
\end{aligned}
$$

Considering the known series sums

$$
\sum_{n \geq 2} t^{n-1}=\frac{t}{1-t} \text { and } \sum_{n \geq 2} n t^{n-1}=\frac{t(2-t)}{(1-t)^{2}}
$$

for $|t|<1$ in the last step, we have

$$
\sum_{n \geq 2}(n-\alpha)\left|\frac{(-1)^{n-1}}{(n-1) ! \rho^{n-1} \prod_{i=1}^{d}\left(\gamma_{i}+1\right)_{n-1}}\right| \leq \frac{4 \rho \mu(2-\alpha)+2(\alpha-1)}{(2 \rho \mu-1)^{2}} \leq 1-\alpha
$$

for $\left|\frac{1}{2 \rho \mu}\right|<1$ and

$$
\alpha \leq \frac{4 \rho^{2} \mu^{2}-12 \rho \mu+3}{4 \rho^{2} \mu^{2}-8 \rho \mu+3} .
$$

So, the proof is completed.

By setting $d=1$ and $\gamma_{1}=\nu$ in the Theorem 2.1, we have the following:

Corollary 2.1. Let $\alpha \in[0,1)$. If

$$
\alpha \leq \frac{64 \nu^{2}+80 \nu+19}{64 \nu^{2}+96 \nu+35}
$$

then the function $z \mapsto F_{\nu}(z)=2^{\nu} z^{1-\frac{\nu}{2}} \Gamma(\nu+1) J_{\nu}(\sqrt{z})$ is starlike of order $\alpha$.

It is well-known from [8, p.13-14] that the basic trigonometric functions can be represented by the classical Bessel function $J_{\nu}$ for the appropriate values of the parameter $\nu$. Clearly, for $\nu=-\frac{1}{2}, \nu=\frac{1}{2}$ and $\nu=\frac{3}{2}$, respectively, we have the following:

$$
J_{-\frac{1}{2}}(z)=\sqrt{\frac{2}{\pi z}} \cos z, J_{\frac{1}{2}}(z)=\sqrt{\frac{2}{\pi z}} \sin z \text { and } J_{\frac{3}{2}}(z)=\sqrt{\frac{2}{\pi z}}\left(\frac{\sin z}{z}-\cos z\right) .
$$

Now, taking $\nu=\frac{1}{2}$ and $\nu=\frac{3}{2}$ in Corollary 2.1 we have the following examples on the elemantary trigonometric functions. 
Example 2.1. The following assertions are true for $z \in \mathbb{D}$ :

a. If $\alpha \leq \alpha_{1} \cong 0.75$, then the function $F_{\frac{1}{2}}(z)=\sqrt{z} \sin \sqrt{z}$ is in $\mathcal{S}^{\star}(\alpha)$.

b. If $\alpha \leq \alpha_{2} \cong 0.88$, then the function $F_{\frac{3}{2}}(z)=3\left(\frac{\sin \sqrt{z}}{\sqrt{z}}-\cos \sqrt{z}\right)$ is in $\mathcal{S}^{\star}(\alpha)$.

Our second main result concerning convexity of order $\alpha$ of the function $F_{\gamma_{d}}(z)$ is the following:

Theorem 2.2. Let $\alpha \in[0,1), d=1,2, \ldots, \rho=(d+1)^{d+1}$ and $\mu=\prod_{i=1}^{d}\left(\gamma_{i}+1\right)$. If

$$
\alpha \leq \frac{(2 \rho \mu-1)^{3}-8 \rho^{2} \mu^{2}+12 \rho \mu-2}{(2 \rho \mu-1)^{3}-16 \rho^{2} \mu^{2}+12 \rho \mu-2},
$$

then the normalized hyper-Bessel function $F_{\gamma_{d}}(z)$ is convex of order $\alpha$ in $\mathbb{D}$.

Proof. By using the Lemma 2.2, it is possible to show that the function $F_{\gamma_{d}}(z)$ is convex of order $\alpha$. For this reason, we need to show that the function $F_{\gamma_{d}}(z)$ satisfies the inequality (2.3). That is, it is sufficient to prove that

$$
\sum_{n \geq 2} n(n-\alpha)\left|\frac{(-1)^{n-1}}{(n-1) ! \rho^{n-1} \prod_{i=1}^{d}\left(\gamma_{i}+1\right)_{n-1}}\right| \leq 1-\alpha .
$$

By making use of the known inequalities (2.5) and (2.6), we can write that

$$
\begin{aligned}
\sum_{n \geq 2} n(n-\alpha)\left|\frac{(-1)^{n-1}}{(n-1) ! \rho^{n-1} \prod_{i=1}^{d}\left(\gamma_{i}+1\right)_{n-1}}\right| & =\sum_{n \geq 2} \frac{n(n-\alpha)}{(n-1) ! \rho^{n-1} \prod_{i=1}^{d}\left(\gamma_{i}+1\right)_{n-1}} \\
& \leq 2 \sum_{n \geq 2} \frac{n(n-\alpha)}{(2 \rho \mu)^{n-1}} \\
& =2 \sum_{n \geq 2} n^{2}\left(\frac{1}{2 \rho \mu}\right)^{n-1}-2 \alpha \sum_{n \geq 2} n\left(\frac{1}{2 \rho \mu}\right)^{n-1} .
\end{aligned}
$$

If we consider the known series sums

$$
\sum_{n \geq 2} n t^{n-1}=\frac{t(2-t)}{(1-t)^{2}} \text { and } \sum_{n \geq 2} n^{2} t^{n-1}=\frac{t\left(t^{2}-3 t+4\right)}{(1-t)^{3}}
$$

for $|t|<1$ in the above last inequality, we get

$$
\begin{aligned}
& \sum_{n \geq 2} n(n-\alpha)\left|\frac{(-1)^{n-1}}{(n-1) ! \rho^{n-1} \prod_{i=1}^{d}\left(\gamma_{i}+1\right)_{n-1}}\right| \leq \frac{8 \rho^{2} \mu^{2}(1-2 \alpha)+2(6 \rho \mu-1)(\alpha-1)}{(2 \rho \mu-1)^{3}} \\
& \text { for }\left|\frac{1}{2 \rho \mu}\right|<1 \text {. Since } \\
& \qquad \frac{8 \rho^{2} \mu^{2}(1-2 \alpha)+2(6 \rho \mu-1)(\alpha-1)}{(2 \rho \mu-1)^{3}} \leq 1-\alpha
\end{aligned}
$$


under asumption, the proof is thus completed.

By setting $d=1$ and $\gamma_{1}=\nu$ in the Theorem 2.2, we have the following:

Corollary 2.2. Let $\alpha \in[0,1)$. If

$$
\alpha \leq \frac{512 \nu^{3}+1216 \nu^{2}+968 \nu+261}{512 \nu^{3}+1088 \nu^{2}+712 \nu+133},
$$

then the function $z \mapsto F_{\nu}(z)=2^{\nu} z^{1-\frac{\nu}{2}} \Gamma(\nu+1) J_{\nu}(\sqrt{z})$ is convex of order $\alpha$.

\section{Hardy space of hyper-Bessel function}

Let $\mathcal{H}$ denote the set of all analytic (holomorphic, regular) functions on the open unit disk $\mathbb{D}$. For any $\eta \in(0, \infty]$, any function $f \in \mathcal{H}$ and any $r \in[0,1)$ set

$$
M_{\eta}(r, f)=\left\{\begin{array}{lr}
\left(\frac{1}{2 \pi} \int_{0}^{2 \pi}\left|f\left(r e^{i \theta}\right)\right|^{\eta} d \theta\right)^{\frac{1}{\eta}}, & \text { if } 0<\eta<\infty \\
\max _{|z| \leq r}|f(z)|, & \text { if } \eta=\infty .
\end{array}\right\}
$$

By definition, the function $f \in \mathcal{H}$ is said to belong to the Hardy space $\mathcal{H}^{\eta}$, where $0<\eta \leq \infty$, if the set $\left\{M_{\eta}(r, f): r \in[0,1)\right\}$ is bounded. Note that for $1 \leq$ $\eta \leq \infty, \mathcal{H}^{\eta}$ is a Banach space with the norm defined by $\|f\|_{\eta}=\lim _{r \rightarrow 1^{-}} M_{\eta}(r, f)$. Furthermore, $\mathcal{H}^{\infty}$ is the class of bounded analytic functions in $\mathcal{H}$. We note that for $0<p \leq q \leq \infty$, it can be shown that $\mathcal{H}^{q}$ is a subset of $\mathcal{H}^{p}$ (see[11]).

The following lemma due to Eenigenburg and Keogh [12] will be used in order derive our last main result.

Lemma 3.1. Let $\alpha \in[0,1)$. If the function $f \in \mathcal{C}(\alpha)$ is not of the form

$$
\left\{\begin{array}{l}
f(z)=k+l z\left(1-z e^{i \theta}\right)^{2 \alpha-1}, \quad \alpha \neq \frac{1}{2} \\
f(z)=k+l \log \left(1-z e^{i \theta}\right), \quad \alpha=\frac{1}{2} .
\end{array}\right\}
$$

for some $k, l \in \mathbb{C}$ and $\theta \in \mathbb{R}$, then the following statements hold:

a. There exist $\delta=\delta(f)>0$ such that $f^{\prime} \in \mathcal{H}^{\delta+\frac{1}{2(1-\alpha)}}$.

b. If $\alpha \in\left[0, \frac{1}{2}\right)$, then there exist $\tau=\tau(f)>0$ such that $f \in \mathcal{H}^{\tau+\frac{1}{1-2 \alpha}}$.

c. If $\alpha \geq \frac{1}{2}$, then $f \in \mathcal{H}^{\infty}$.

Our main result concerning the Hardy space of the normalized hyper-Bessel function $F_{\gamma_{d}}(z)$ is as follows.

Theorem 3.1. Let $\alpha \in[0,1), d=1,2, \ldots, \rho=(d+1)^{d+1}$ and $\mu=\prod_{i=1}^{d}\left(\gamma_{i}+1\right)$. If

$$
\alpha \leq \frac{(2 \rho \mu-1)^{3}-8 \rho^{2} \mu^{2}+12 \rho \mu-2}{(2 \rho \mu-1)^{3}-16 \rho^{2} \mu^{2}+12 \rho \mu-2},
$$

then the normalized hyper-Bessel function $F_{\gamma_{d}}(z)$ has the following properties: 
i. $F_{\gamma_{d}}(z) \in \mathcal{H}^{\frac{1}{1-2 \alpha}}$ for $\alpha \in\left[0, \frac{1}{2}\right)$,

ii. $F_{\gamma_{d}}(z) \in \mathcal{H}^{\infty}$ for $\alpha \in\left(\frac{1}{2}, 1\right)$.

Proof. Gauss hypergeometric function is defined by

$$
{ }_{2} F_{1}(a, b, c ; z)=\sum_{n \geq 0} \frac{(a)_{n}(b)_{n}}{(c)_{n}} \frac{z^{n}}{n !} .
$$

By using the definetion of the Gauss hypergeometric function given by (3.1), it is easily shown that

$$
\begin{aligned}
k+\frac{l z}{\left(1-z e^{i \theta}\right)^{1-2 \alpha}} & =k+l z_{2} F_{1}\left(1,(1-2 \alpha), 1 ; z e^{i \theta}\right) \\
& =k+l \sum_{n \geq 0} \frac{(1-2 \alpha)_{n}}{n !} e^{i \theta n} z^{n+1}
\end{aligned}
$$

for $k, l \in \mathbb{C}, \alpha \neq \frac{1}{2}$ and $\theta \in \mathbb{R}$. On the other hand, it can be easily shown that

$$
\begin{aligned}
k+l \log \left(1-z e^{i \theta}\right) & =k-l z_{2} F_{1}\left(1,1,2 ; z e^{i \theta}\right) \\
& =k-l \sum_{n \geq 0} \frac{1}{n+1} e^{i \theta n} z^{n+1} .
\end{aligned}
$$

As a result, the function $F_{\gamma_{d}}(z)$ given by $(2.4)$ is not of the forms $k+\frac{l z}{\left(1-z e^{i \theta}\right)^{1-2 \alpha}}$ for $\alpha \neq \frac{1}{2}$ and $k+l \log \left(1-z e^{i \theta}\right)$ for $\alpha=\frac{1}{2}$, respectively. Also, it is known from Theorem 2.2 that, the function $F_{\gamma_{d}}(z)$ is convex of order $\alpha$. Hence, by Lemma 3.1 the proof is completed.

By setting $d=1$ and $\gamma_{1}=\nu$ in the Theorem 3.1, we have the following:

Corollary 3.1. Let $\alpha \in[0,1)$. If

$$
\alpha \leq \frac{512 \nu^{3}+1216 \nu^{2}+968 \nu+261}{512 \nu^{3}+1088 \nu^{2}+712 \nu+133}
$$

then the function $z \mapsto F_{\nu}$ has the following properties:

i. $F_{\nu}(z) \in \mathcal{H}^{\frac{1}{1-2 \alpha}}$ for $\alpha \in\left[0, \frac{1}{2}\right)$,

ii. $F_{\nu}(z) \in \mathcal{H}^{\infty}$ for $\alpha \in\left(\frac{1}{2}, 1\right)$. 


\section{REF E R E N C E S}

1. İ. AKTAŞ: Partial sums of hyper-Bessel function with applications. Hacet. J. Math. Stat. (In press).

2. I. AKTAŞ: On some properties of hyper-Bessel and related functions. TWMS J. App. and Eng. Math. 9 (1) (2019), 30-37.

3. İ. AKTAŞ and Á. BARICZ: Bounds for the radii of starlikeness of some q-Bessel functions. Results Math. 72 (1-2) (2017), 947-963.

4. İ. AKtAŞ, Á. BARICZ and H. ORHAN: Bounds for the radii of starlikeness and convexity of some special functions. Turk. J. Math. 42 (1) (2018), 211-226.

5. İ. AKtAŞ, Á. BARICZ and S. Singh: Geometric and monotonic properties of hyper-Bessel functions. Ramanujan. J. doi.org/10.1007/s11139-018-0105-9

6. İ. AKTAŞ, Á. BARICZ and N. YAĞMUR: Bounds for the radii of univalence of some special functions. Math. Inequal. Appl. 20 (3) (2017), 825-843.

7. Á. BARICZ: Bessel transforms and Hardy space of generalized Bessel functions. Mathematica 48 (2006), 127-136.

8. Á. BARICZ: Generalized Bessel Functions of the First Kind. Springer-Verlag, Berlin, 2010.

9. R. K. Brown: Univalence of Bessel functions. Proc. Amer. Math. Soc. 11 (2) (1960), 278-283.

10. P. Delerue: Sur le calcul symbolic à $n$ variables et fonctions hyper-besséliennes (II). Annales Soc. Sci. Bruxelle Ser.1, 3 (1953), 229-274.

11. P. L. Duren: Theory of $\mathcal{H}^{p}$ spaces. Academic Press, Newyork, 1970.

12. P. J. Eenigenburg and F. R. KeOGH: The Hardy class of some univalent functions and their derivatives. Michigan Math. J. 17 (1970), 335-346.

13. E. KReYszig and J. TodD: The radius of univalence of Bessel functions. Illinois J. Math. 4 (1960), 143-149.

14. S. Ponnusamy: The Hardy space of hypergeometric functions. Complex Var. Elliptic., 29 (1996), 83-96.

15. H. Silverman: Univalent functions with negative coefficients. Proc. Am. Math. Soc. 51 (1) (1975), 109-116.

16. G. N. Watson: A Treatise of the Theory of Bessel Functions. Cambridge Univ. Press, Cambridge, 1944.

İbrahim Aktaş

Karamanoğlu Mehmetbey University

Kamil Özdağ Science Faculty

Department of Mathematics

70100 Karaman, Turkey

aktasibrahim38@gmail .com 